\title{
EFFECTS OF HEAT AND MASS FLUX ON MHD FREE CONVECTION FLOW THROUGH A POROUS MEDIUM WITH RADIATION AND FIRST ORDER CHEMICAL REACTION
}

\author{
B. AWASTHI \\ Department of Mathematics, JECRC University \\ Jaipur -303905, Rajasthan, INDIA \\ E-mail: bhavtosh123@gmail.com
}

\begin{abstract}
The study of a magneto hydrodynamic (MHD) free convection flow of an incompressible viscous fluid flow past a vertical surface is analyzed by taking into account viscous dissipation under the influence of radiation effect and chemical reaction with constant heat and mass fluxes. The governing partial differential equations have been converted into a set of ordinary differential equations using non dimensional quantities. The perturbation technique has been applied to solve the system of partial differential equations. The velocity, temperature and concentration fields have been studied for the effect of the permeability parameter $(\alpha)$, Grashof number for heat transfer (Gr), Grashof number for mass transfer (Gm), Prandtl number $(\mathrm{Pr})$, magnetic parameter $(M)$, chemical reaction parameter $(\mathrm{Kr})$, Schmidt number $(\mathrm{Sc})$, Eckert number $(\mathrm{E})$, radiation parameter $(F)$ etc. The expressions for the skin-friction, rate of heat transfer and rate of mass transfer are also derived. It is observed that when the radiation parameter increases, the velocity increases near the vertical surface. It is also seen that the concentration decreases with an increase in the chemical reaction parameter $(K r)$.
\end{abstract}

Key words: first order chemical reaction, porous medium, viscous dissipation, MHD, radiation, heat and mass fluxes.

\section{Introduction}

In many industrial and real life problems, there exist flows which are stimulated not only by the differences of temperature but by differences of concentration also. These mass transfer differences affect the rate of heat transfer. In industries, lot of transport processes exist in which heat and mass transfer takes place. The phenomenon of heat and mass transfer frequently exists in many chemical processing industries such as food processing and polymer process. Free convection flows are of great interest in many industrial applications such as fiber and granular insulation, geothermal systems, etc.

Magnetohydrodynamics (MHD) is the science, which deals with the motion of an electrically conducting fluid in the presence of magnetic fields. The study of magnetohydrodynamics with heat and mass transfer in the presence of radiation has attracted many researchers and engineers due to diverse applications in different areas of science and technology. MHD plays an important role in geophysics, agriculture, petroleum industries, astrophysics, geological formations, in exploration and thermal recovery of oil, geothermal reservoirs and underground nuclear waste storage sites. In the field of power generation, MHD is receiving considerable attention due to the possibilities it offers for much higher thermal efficiencies in power plants. Lin and $\mathrm{Wu}$ [1] studied simultaneous heat and mass transfer in laminar free convection boundary-layer flow along a vertical plate. Chamka [2] investigated the MHD flow past a uniformly stretched vertical permeable surface in the presence of heat generation / absorption and a chemical reaction. Chamkha [3] studied the unsteady MHD convective heat and mass transfer over a semi-infinite vertical permeable moving plate with heat absorption. Chaudhary et al. [4] analyzed the effect of radiation on heat transfer in MHD mixed convection flow and mass transfer past an infinite vertical plate with Ohmic heating 
and viscous dissipation. He derived the solution for the velocity, temperature and concentration profiles. Chaudhary and Jha [5] investigated an unsteady hydromagnetic free convection flow of an elastic viscoelastic fluid past an infinite vertical plate when the temperature and concentration are assumed to be oscillating with time in the presence of Hall effects.

The effect of viscous dissipation plays an important role in natural convection in various devices that are subjected to large deceleration or which operate at high rotational speeds. Natural convection flow is often encountered in cooling of nuclear reactors or in the study of the structure of stars and planets. The study of temperature and heat transfer is of great importance to engineers because of its almost universal occurrence in many branches of science and engineering. The viscous dissipation effects are also important in geophysical flows and in certain industrial operations and are usually characterized by the Eckert number. Soundalgekar [6] analyzed unsteady free convective flow past an infinite vertical porous plate when the temperature oscillates in time and with constant suction at the plate. Cortel [7] analyzed the effect of viscous dissipation in the MHD flow and heat transfer of a viscoelastic fluid over a stretching sheet. Kumar [8] studied the effects of radiation and viscous dissipation over a stretching surface subjected to variable heat flux in the presence of a transverse magnetic field. Kishan and Amrutha [9] discussed a two-dimensional steady nonlinear MHD boundary layer flow of an incompressible, viscous, electrically conductive and Bousinesque fluid flowing over a vertical stretching surface in the presence of a uniform magnetic field by taking into account the viscous dissipation with heat, mass transfer, chemical reaction and thermal stratification effects. The combined effects of viscous dissipation and heat generation on an MHD natural convection flow along a vertical wavy surface were studied by Sujon and Parveen [10].

The effect of radiation on an MHD flow has become very important in many industrial applications. A large number of engineering processes takes place at high temperatures and so the knowledge of radiation and heat transfer is essential for designing various types of industrial equipment. Nuclear power plants, gas turbines and various propulsion devices for different type of aircrafts, missiles and satellites are some important examples of such processes. Hossain et al. [11] analyzed the effect of radiation on free convection from a porous vertical plate. Chamkha [2] studied thermal radiation and buoyancy effects on a hydromagnetic flow over an accelerating permeable surface with heat source or sink. He described the effects of various parameters on the velocity and temperature. The combined free convective boundary-layer flow with the thermal radiation effect and mass transfer past a moving vertical porous plate, when the plate moves in its own plane was studied by Makinde [12]. The radiation effects on an unsteady flow of a viscous incompressible fluid past an exponentially accelerated infinite isothermal vertical plate with uniform mass diffusion in the presence of a magnetic field and heat source were studied by Kulkarni and Patil [13].

Chemical reactions can be either heterogeneous or homogeneous processes. It depends on whether they occur at an interface or as a single phase volume reaction. The reaction is heterogeneous if it takes place at an interface level and homogeneous if it takes place in solution. In most chemical reactions, the reaction rate depends on the concentration of the species. A reaction is said to be of first order, if the rate of reaction is directly proportional to the concentration itself. In many chemical engineering processes, a chemical reaction occurs between a foreign mass and the fluid in which the plate is moving. These processes take place in many industrial applications, e.g. polymer production, manufacturing of ceramics or glassware and food processing. The first order chemical reaction in the neighborhood of a horizontal plate was examined by Chambre and Young [14]. Chen and Yuh [15] dealt with steady combined heat and mass transfer effects for both conditions of uniform wall temperature/concentration and uniform heat/mass flux. Das et al. [16] investigated the effect of first order homogeneous chemical reaction on the flow past an infinite vertical plate with uniform heat and mass fluxes. Muthucumarswamy [17] analyzed the chemical reaction effect with thermal stratification over a vertical stretching surface. The effects of a chemical reaction, thermophoresis and variable viscosity on a hydro magnetic flow with heat and mass transfer over a flat plate in the presence of heat generation/absorption were examined by Seddeek [18]. Raptis and Perdikis [19] studied the effect of the magnetic field and chemical reaction on a viscous flow over a non linear stretching sheet. Kabeir and Abdou [20] analyzed the effect of chemical reaction on a micro polar flow over an isothermal vertical cone. The effect of a chemical reaction and radiation absorption on a free convection flow through a porous medium with variable suction in the presence of a uniform magnetic field was investigated by Sudheer Babu 
and Satyanarayana [21]. Mahapatra et al. [22] studied the effect of a chemical reaction on a free convection flow through a porous medium bounded by a vertical infinite surface. He observed that the velocity and concentration increase during a generative reaction and decrease in a destructive reaction. Chandra Shekar and Kishan [23] studied the unsteady magnetohydrodynamic convective flow of heat and mass transfer of a viscous, incompressible, electrically conducting Newtonian fluid along a vertical permeable plate in the presence of a homogeneous first order chemical reaction and thermal radiation effects. The unsteady free convection heat and mass transfer flow through a non-homogeneous porous medium with variable permeability bounded by an infinite porous vertical plate in slip flow regime in the presence of thermal radiation and chemical reaction was studied by Ibrahim and Suneetha [24].

When a chemical reaction takes place in a process, a large amount of heat is generated .Due to this, the temperature of the body increases. To maintain the temperature of the body insulation is required. Porous media are widely used to insulate a heated body to maintain its temperature during the process. To insulate the surface of the body in which the process takes place more effectively, it is necessary to study the flow through a porous medium and to estimate the effect of chemical reaction on mass and heat transfers. Yamamoto et al. [25] analyzed acceleration of convection in a porous permeable medium along an arbitrary smooth surface. Raptis and Singh [26] discussed a flow past an impulsively vertical plate in a porous medium by a finite difference method. Sattar et al. [27] studied an unsteady free convection flow along a vertical porous plate embedded in a porous medium. The thermal radiation effect on an unsteady MHD flow past a vertical porous plate immersed in a porous medium was analyzed by Samad and Rahman [28]. Sharma and Singh [29] discussed an unsteady MHD free convective flow and heat transfer along a vertical porous plate with variable suction and internal heat generation. Sharma et al. [30] analyzed the heat and mass transfer effects on an unsteady MHD free convective flow along a vertical porous plate with internal heat generation and variable suction.

The above studies did not consider the heat and mass transfer effect on the steady free convection flow over a vertical surface in the presence of radiation absorption, chemical reaction and uniform heat and mass flux. So, the aim of the present work is to study the heat and mass transfer effect on the steady free convection flow over a vertical surface in the presence of radiation, chemical reaction under the influence of uniform heat and mass flux.

\section{Mathematical formulation of the problem}

In the present work, we study a two dimensional flow of a viscous incompressible, electrically conducting fluid through a porous medium occupying semi-infinite region of space bounded by a vertical infinite surface. A uniform magnetic field has been applied normal to the direction of the flow. Here we assume that a chemically reactive species is emitted from the surface and diffuses into the fluid. The $x^{*}$ axis is chosen along the plate and the $y^{*}$ axis is perpendicular to it. The induced magnetic field is neglected under the assumption that the magnetic Reynolds number is small. The reaction is assumed to take place entirely in the stream.

The basic equations for the flow from porous media are

$$
\begin{aligned}
& \frac{\partial v^{*}}{\partial y^{*}}=0, \\
& v^{*} \frac{\partial u^{*}}{\partial y^{*}}=v \frac{\partial^{2} u^{*}}{\partial y^{* 2}}+g \beta_{1}\left(T^{*}-T_{\infty}\right)+g \beta_{2}\left(C^{*}-C_{\infty}\right)-\frac{v u^{*}}{k}-\frac{\sigma B_{0}^{2} u^{*}}{\rho},
\end{aligned}
$$




$$
\begin{aligned}
& v^{*} \frac{\partial T^{*}}{\partial y^{*}}=\frac{\lambda}{\rho C_{p}} \frac{\partial^{2} T^{*}}{\partial y^{* 2}}+\frac{v}{C_{p}}\left(\frac{\partial u^{*}}{\partial y^{*}}\right)^{2}-\frac{1}{\rho C_{p}} \frac{\partial q_{r}}{\partial y^{*}}, \\
& v^{*} \frac{\partial C^{*}}{\partial y^{*}}=D \frac{\partial^{2} C^{*}}{\partial y^{* 2}}-k_{c} C^{*} .
\end{aligned}
$$

We assume that the level of species concentration is very low. Therefore the heat generated due to the chemical reaction is neglected.

From Eq.(2.1), we get

$$
v^{*}=\text { constant }=-v_{0}(\text { assume })
$$

where $v_{0}$ is constant suction velocity normal to the surface.

Cogley et al. [31] showed that the radiative heat flux is given by

$$
\frac{\partial q_{r}}{\partial y^{*}}=4\left(T^{*}-T_{\infty}\right) I
$$

where $I=\int K_{\lambda} \frac{\partial e_{\lambda}}{\partial T^{*}} d \lambda, K_{\lambda}$ is the absorption coefficient at the plate and $e_{\lambda}$ is Plank's function.

The relevant boundary conditions are

$$
\begin{aligned}
& u^{*}=0, \quad T^{*}=T_{\infty}, \quad C^{*}=C_{\infty} \quad \text { for any value of } y^{*}, \quad t \leq 0, \\
& u^{*}=0, \quad \frac{\partial T^{*}}{\partial y^{*}}=-\frac{q}{\lambda}, \quad \frac{\partial C^{*}}{\partial y^{*}}=-\frac{m}{D}, \quad y^{*}=0, \quad t>0, \\
& u^{*} \rightarrow 0, \quad T^{*} \rightarrow T_{\infty}, \quad C^{*} \rightarrow C_{\infty}, \quad y^{*} \rightarrow \infty, \quad t>0 .
\end{aligned}
$$

Using Eq.(2.5), Eqs (2.1), (2.2), (2.3) and (2.4) reduce to

$$
\begin{aligned}
& -v_{0} \frac{\partial u^{*}}{\partial y^{*}}=v \frac{\partial^{2} u^{*}}{\partial y^{* 2}}+g \beta_{1}\left(T^{*}-T_{\infty}\right)+g \beta_{2}\left(C^{*}-C_{\infty}\right)-\frac{v u^{*}}{k}-\frac{\sigma B_{0}^{2} u^{*}}{\rho}, \\
& -v_{0} \frac{\partial T^{*}}{\partial y^{*}}=\frac{\lambda}{\rho C_{p}} \frac{\partial^{2} T^{*}}{\partial y^{* 2}}+\frac{v}{C_{p}}\left(\frac{\partial u^{*}}{\partial y^{*}}\right)^{2}-\frac{1}{\rho C_{p}} \frac{\partial q_{r}}{\partial y^{*}}, \\
& -v_{0} \frac{\partial C^{*}}{\partial y^{*}}=D \frac{\partial^{2} C^{*}}{\partial y^{* 2}}-k_{c} C^{*} .
\end{aligned}
$$

Let us introduce the following non-dimensional variables 


$$
\begin{aligned}
& f(\eta)=\frac{u^{*}}{v_{0}}, \quad \eta=\frac{v_{0} y^{*}}{v}, \quad \operatorname{Pr}=\frac{v \rho C_{p}}{\lambda}, \quad F=\frac{4 v^{2} I}{\lambda v_{0}^{2}}, \quad \alpha=\frac{v_{0}^{2} k}{v^{2}}, \\
& C=\frac{\left(C^{*}-C_{\infty}\right) D}{m}, \quad \mathrm{Gr}=\frac{g \beta_{1} q v}{\lambda v_{0}{ }^{3}}, \quad \mathrm{Gm}=\frac{g \beta_{2} m v}{D v_{0}{ }^{3}}, \quad \mathrm{Sc}=\frac{v}{D}, \\
& \theta=\frac{\left(T^{*}-T_{\infty}\right) \lambda}{v q}, \quad K r=\frac{k_{c} v}{v_{0}^{2}}, \quad E=\frac{\lambda v_{0}^{2}}{q c_{p}}, \quad M=\frac{\sigma v B_{0}^{2}}{\rho v_{0}^{2}} .
\end{aligned}
$$

Substituting Eq. (2.11) into Eqs. (2.8) - (2.10), we get the following dimensionless equations

$$
\begin{aligned}
& f^{\prime \prime}+f^{\prime \prime}-\left(\frac{1}{\alpha}+M\right) f=-\theta-C, \\
& \theta^{\prime \prime}+\theta^{\prime}-F \theta=-E\left(f^{\prime}\right)^{2}, \\
& C^{\prime \prime}+\operatorname{Sc} C^{\prime}=\operatorname{Sc} k_{r} C .
\end{aligned}
$$

The corresponding boundary conditions are

$$
\begin{gathered}
\eta=0, \quad f=0, \quad \theta^{\prime}=-1, \quad C=-1, \\
\eta \rightarrow \infty, \quad f \rightarrow 0, \quad \theta \rightarrow 0, \quad C \rightarrow 0 .
\end{gathered}
$$

\section{Solution of the problem}

To obtain the solution of the coupled non-linear system of Eqs (2.12)-(2.14) with the boundary conditions (2.15), we assume the solution in the neighborhood of the plate in terms of increasing powers of E. Expand $f, \theta$ and $C$ in powers of the Eckert number $\mathrm{E}$ and assume that it is very small. This is justified in a low speed incompressible flow. Hence we get

$$
\begin{aligned}
& f=f_{0}+\mathrm{E} f_{1}+O\left(E^{2}\right), \\
& \theta=\theta_{0}+\mathrm{E} \theta_{1}+O\left(E^{2}\right), \\
& C=C_{0}+\mathrm{E} C_{1}+O\left(E^{2}\right) .
\end{aligned}
$$

Substituting Eq. (3.1) into Eqs. (2.12)-(2.14) and equating like powers of E and neglecting higher order terms in $\mathrm{E}$, we get

$$
f_{0}^{\prime \prime}+f_{0}^{\prime}-\delta f_{0}=-\mathrm{Gr} \theta_{0}-\mathrm{GmC}_{0},
$$




$$
\begin{aligned}
& f_{1}^{\prime \prime}+f_{1}^{\prime}-\delta f_{1}=-\mathrm{Gr} \theta_{1}-\mathrm{GmC}_{1}, \\
& \theta_{0}^{\prime \prime}+\operatorname{Pr} \theta_{0}^{\prime}-F \theta_{0}=0, \\
& \theta_{1}^{\prime \prime}+\theta_{1}^{\prime}-F \theta_{1}=-f_{0}^{\prime 2}, \\
& C_{0}^{\prime \prime}+\operatorname{Sc} C_{0}^{\prime}=\operatorname{Sc} k_{r} C_{0}, \\
& \mathrm{C}_{1}^{\prime \prime}+\operatorname{Sc} C_{1}^{\prime}=\operatorname{Sc} k_{r} C_{1},
\end{aligned}
$$

and the corresponding boundary conditions are

$$
\begin{aligned}
& f_{0}=0, \quad f_{1}=0, \quad \theta_{0}{ }^{\prime}=-1, \quad \theta_{1}^{\prime}=0, \quad C_{0}{ }^{\prime}=-1, \quad C_{1}^{\prime}=0 \text { at } \eta=0, \\
& f_{0} \rightarrow 0, f_{1} \rightarrow 0, \theta_{0} \rightarrow 0, \theta_{1} \rightarrow 0, \quad C_{0} \rightarrow 0, \quad C_{1} \rightarrow 0 \text { at } \eta \rightarrow \infty .
\end{aligned}
$$

Solving Eqs (3.1)-(3.6) under the boundary conditions (3.7), we get

$$
\begin{aligned}
& f_{0}=K_{3} e^{h_{2} \eta}+K_{1} e^{e_{2} \eta}+K_{2} e^{d_{2} \eta} \\
& f_{1}=K_{18} e^{h_{2} \eta}+K_{11} e^{e_{2} \eta}+K_{12} e^{2 h_{2} \eta}+K_{13} e^{2 e_{2} \eta}+ \\
& +K_{14} e^{2 d_{2} \eta}+K_{15} e^{\left(h_{2}+e_{2}\right) \eta}+K_{16} e^{\left(d_{2+} e_{2}\right) \eta}+K_{17} e^{\left(d_{2+} h_{2}\right) \eta}, \\
& \theta_{0}=-\frac{1}{e_{2}} e^{e_{2} \eta} \\
& \theta_{1}=K_{10} e^{e_{2} \eta}+K_{4} e^{2 h_{2} \eta}+K_{5} e^{2 e_{2} \eta}+K_{6} e^{2 d_{2} \eta}+K_{7} e^{\left(h_{2+} e_{2}\right) \eta}+K_{8} e^{\left(d_{2+} e_{2}\right) \eta}+K_{9} e^{\left(d_{2+} h_{2}\right) \eta}, \\
& C_{0}=-\frac{1}{d_{2}} e^{d_{2} \eta} \\
& \mathrm{C}_{1}=0 .
\end{aligned}
$$

Substituting the solutions of Eqs (3.9)-(3.14) in Eq. (3.1), we obtain

$$
\begin{aligned}
& f(\eta)=K_{3} e^{h_{2} \eta}+K_{1} e^{e_{2} \eta}+K_{2} e^{d_{2} \eta}+E\left(K_{18} e^{h_{2} \eta}+K_{11} e^{e_{2} \eta}+K_{12} e^{2 h_{2} \eta}+\right. \\
& \left.+K_{13} e^{2 e_{2} \eta}+K_{14} e^{2 d_{2} \eta}+K_{15} e^{\left(h_{2+} e_{2}\right) \eta}+K_{16} e^{\left(d_{2+} e_{2}\right) \eta}+K_{17} e^{\left(d_{2+} h_{2}\right) \eta}\right), \\
& \theta(\eta)=-\frac{1}{e_{2}} e^{e_{2} \eta}+E\left(K_{10} e^{e_{2} \eta}+K_{4} e^{2 h_{2} \eta}+K_{5} e^{2 e_{2} \eta}+K_{6} e^{2 d_{2} \eta}+\right. \\
& \left.+K_{7} e^{\left(h_{2+} e_{2}\right) \eta}+K_{8} e^{\left(d_{2+} e_{2}\right) \eta}+K_{9} e^{\left(d_{2+} h_{2}\right) \eta}\right),
\end{aligned}
$$




$$
C(\eta)=-\frac{1}{d_{2}} e^{d_{2} \eta}
$$

The rate of heat transfer in terms of the Nusselt number is given by

$$
\begin{aligned}
& \mathrm{Nu}=-\left(\frac{\partial \theta}{\partial \eta}\right)_{\eta=0}=1-E\left(e_{2} K_{10}+2 h_{2} K_{4}+2 e_{2} K_{5}+2 d_{2} K_{6}+\right. \\
& \left.+\left(h_{2+} e_{2}\right) K_{7}+\left(d_{2+} e_{2}\right) K_{8}+\left(d_{2}+h_{2}\right) K_{9}\right) .
\end{aligned}
$$

The non-dimensional skin friction at the surface is given by

$$
\begin{aligned}
& \tau=\left(\frac{\partial \mathrm{f}}{\partial \eta}\right)_{\eta=0}=K_{3} h_{2}+K_{1} e_{2}+K_{2} d_{2}+E\left[K_{18} h_{2}+K_{11} e_{2}+2 K_{12} h_{2}+2 K_{13} e_{2}+\right. \\
& +2 K_{14} d_{2}+2 d_{2} K_{14}+K_{15}\left(\left(e_{2+} h_{2}\right)+K_{16}\left(\left(d_{2+} e_{2}\right)+K_{17}\left(\left(d_{2+} h_{2}\right)\right] .\right.\right.
\end{aligned}
$$

The rate of mass transfer in terms of the Sherwood number (Sh) is given by

$$
\mathrm{Sh}=\left(\frac{\partial C}{\partial \eta}\right)_{\eta=0}=-1+\mathrm{E}(-1)=-(1+\mathrm{E})
$$

\section{Results and discussion}

In order to get a clear picture of the physical problem, numerical results are presented in figures to study the effects of radiation, heat flux and mass flux on the free MHD convection flow through a porous medium bounded by a vertical surface in the presence of a chemical reaction for different values of the permeability parameter $(\alpha)$, Grashof number for heat transfer (Gr), modified Grashof number (Gm), Prandtl number $(\mathrm{Pr})$, magnetic parameter $(M)$, chemical reaction parameter $(\mathrm{Kr})$, Schmidt number $(\mathrm{Sc})$, Eckert Number (E), radiation parameter $(F)$ etc.

Velocity profiles: The velocity profiles for different parameters are depicted in Figs 1-6. Velocity profiles for different values of the chemical reaction parameter are presented in Fig.1. It is noticed that velocity decreases with an increase in the chemical reaction parameter. Figure 2 shows the effect of the permeability parameter $(\alpha)$ on velocity profiles. It is observed that velocity increases with an increase in the permeability parameter. It is interesting to observe that the fluid velocity increases and reaches its maximum over a very short distance from the plate and then decreases gradually to zero for a positive value of $\alpha$. The velocity profile for different values of the radiation parameter $(F)$ is depicted in Fig.3. It is observed that the velocity increases with an increase in the radiation parameter. The effect of the Prandtl number Pr on the velocity profile is depicted in Fig.4. It is observed that velocity increases with an increase in the Prandtl number ( $\operatorname{Pr}$ ). Figure 5 shows the effect of the Grashof number for mass transfer $(\mathrm{Gm})$ on the velocity profile. An increase in Gm results in a decrease in velocity. Figure 6 shows the effect of the Grashof number for heat transfer (Gr) on the velocity profile. An increase in Gr results in an increase in velocity. 


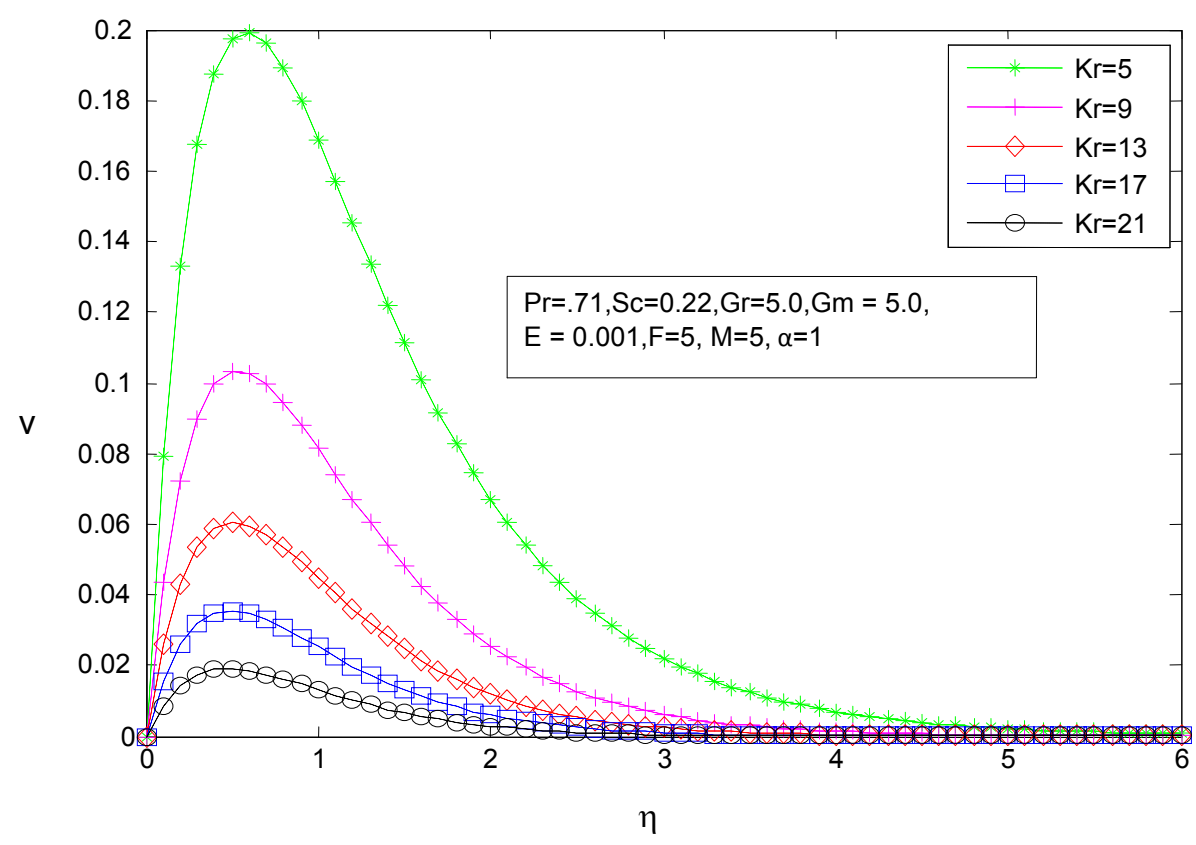

Fig.1. Velocity profile for different values of the chemical reaction parameter $(K r)$.

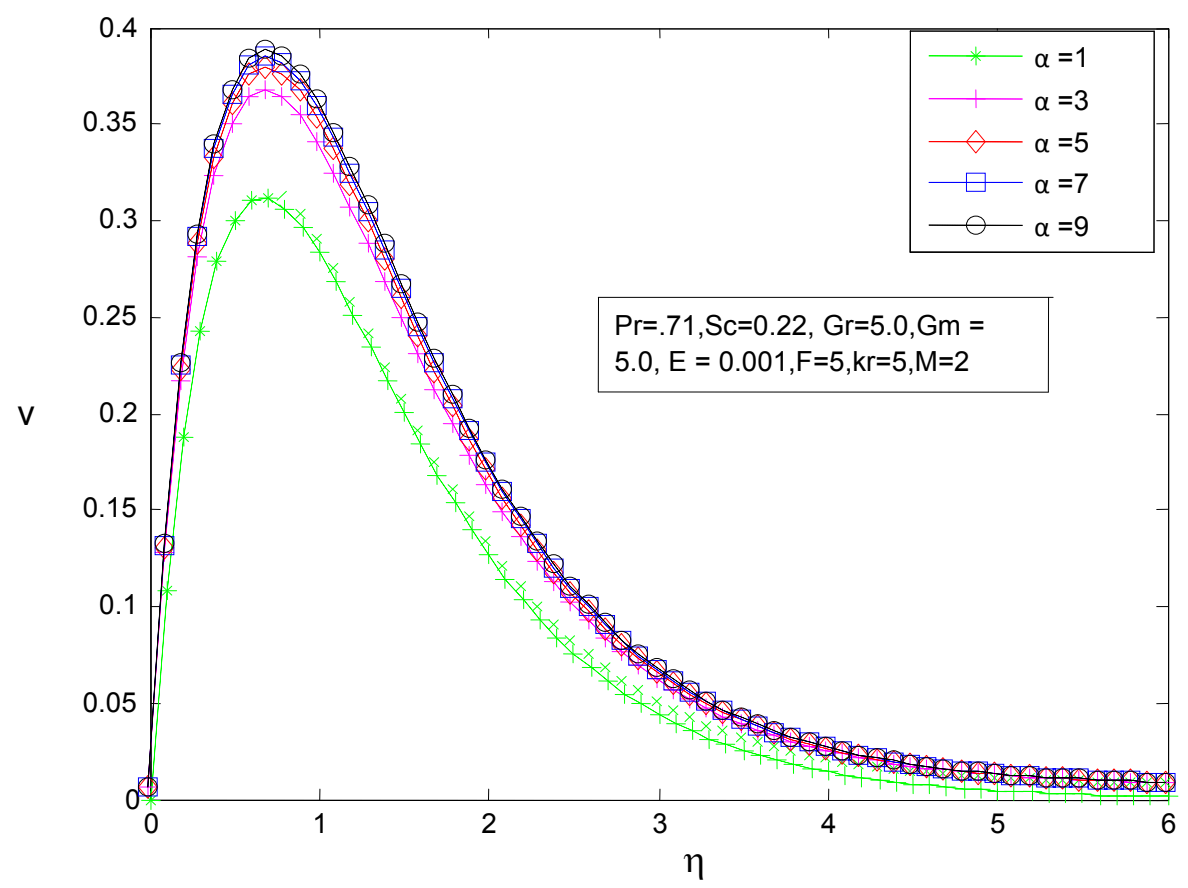

Fig.2. Velocity profile for different values of the permeability parameter $(\alpha)$. 


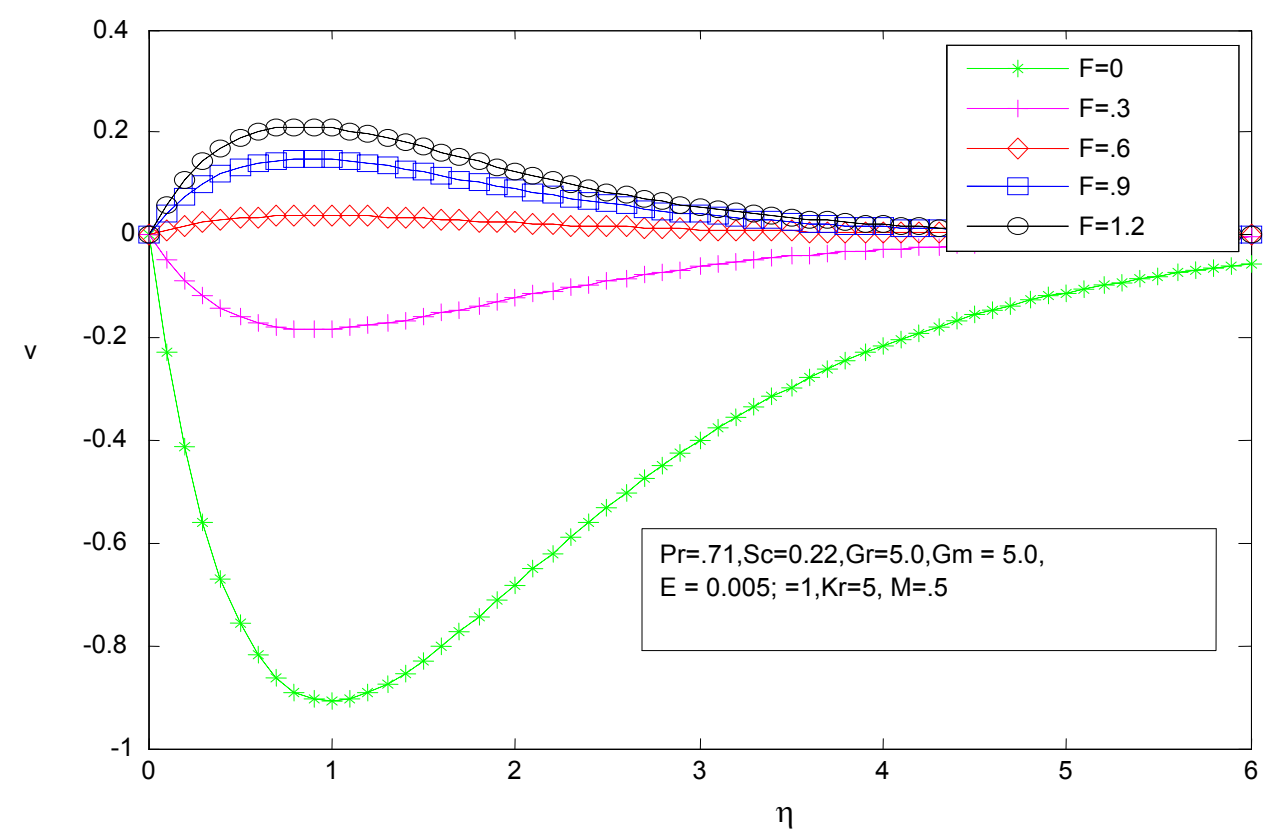

Fig.3. Velocity profile for different values of the radiation parameter $(F)$.

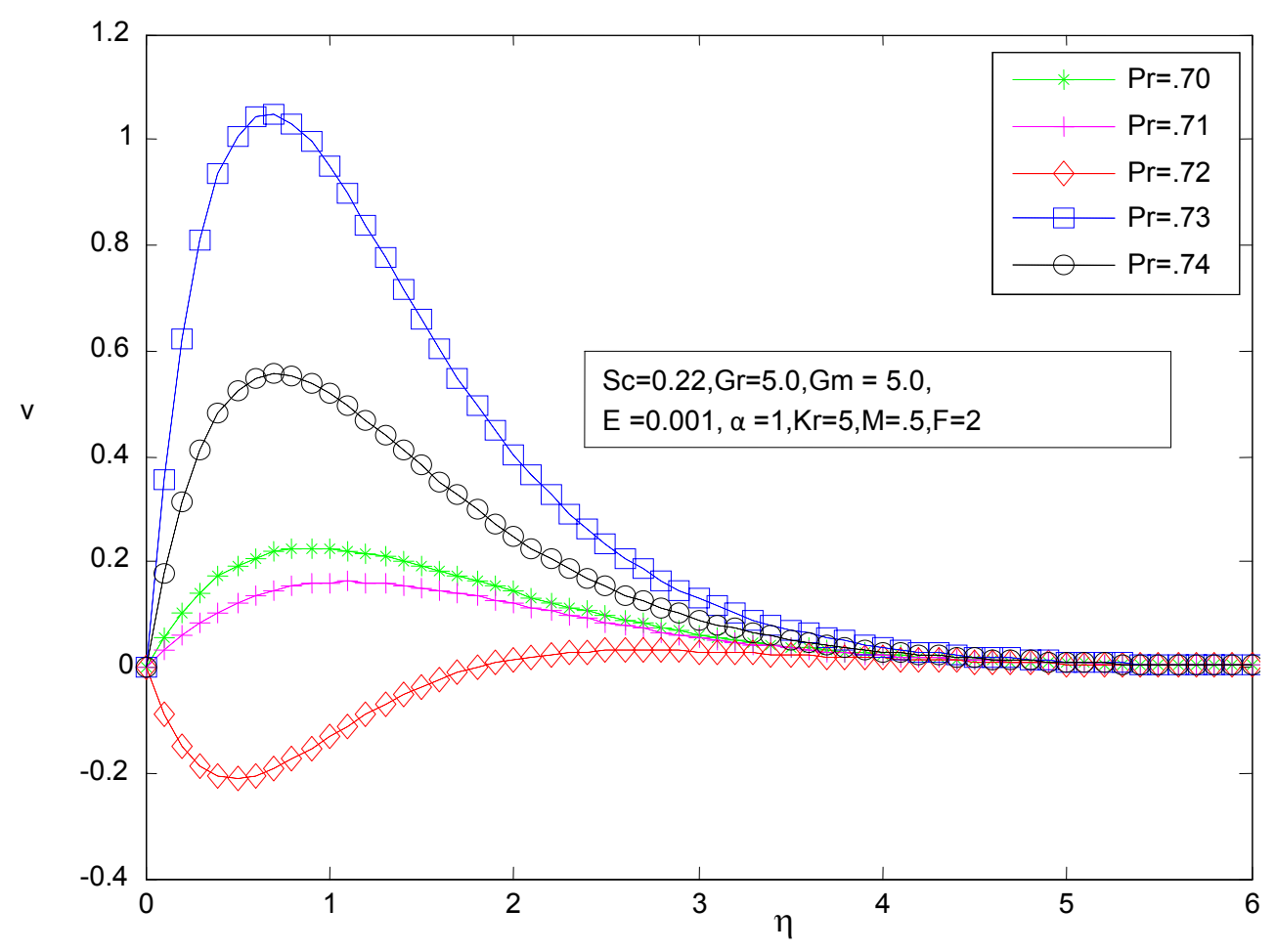

Fig.4. Velocity profile for different values of the Prandtl number (Pr). 


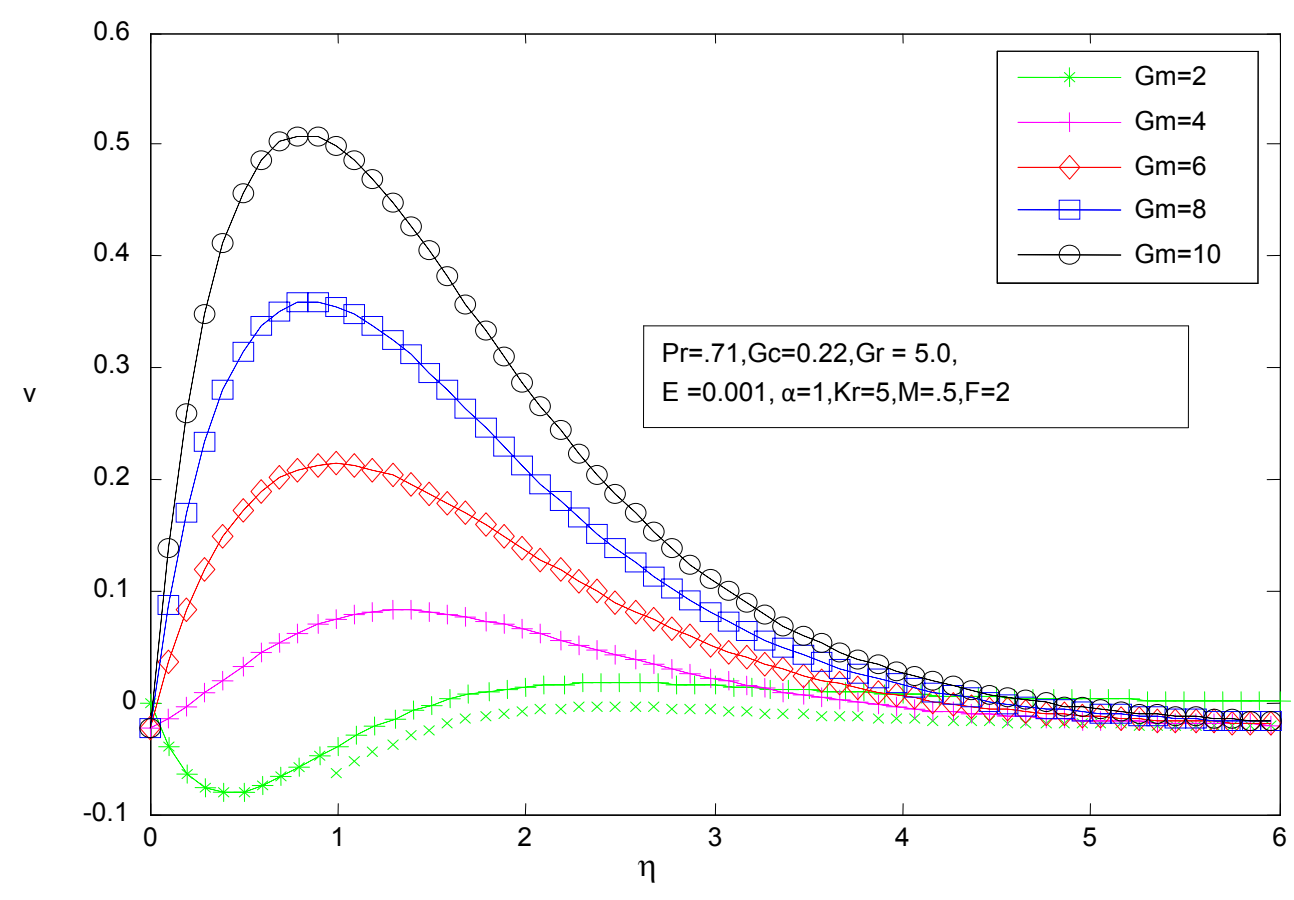

Fig.5. Velocity profile for different values of the $(\mathrm{Gm})$ Grashof number for mass transfer.

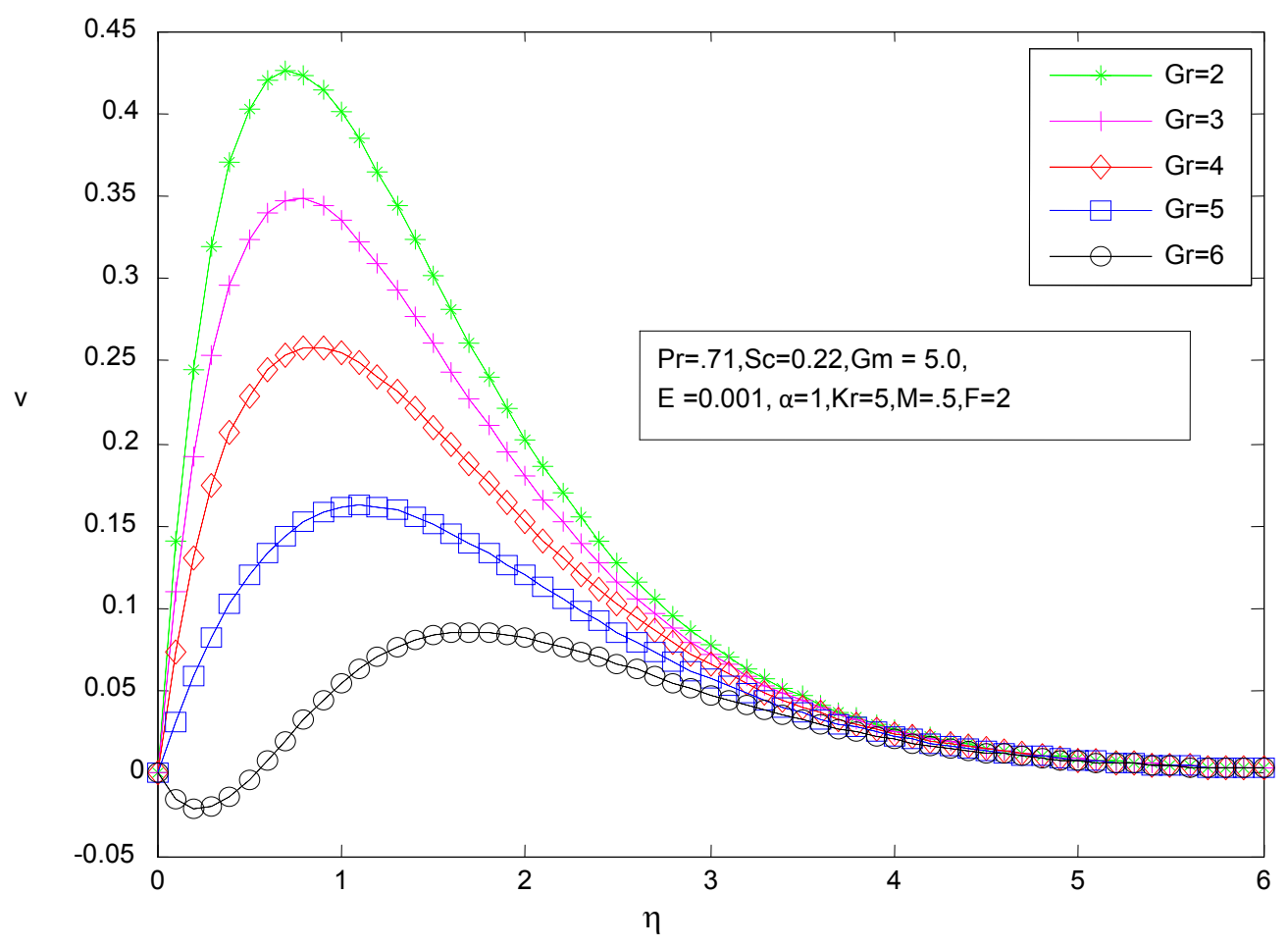

Fig.6. Velocity profile for different values of the (Gr) Grashof number for heat transfer.

Temperature profiles: The temperature profiles are depicted in Figs 7-10. In Fig.7, the influence of the Eckert Number ( E ) on temperature is shown. It is seen that the temperature increases with an increase in the Eckert number (E). Figure 8 displays the effects of the Grashof number for mass transfer $(\mathrm{Gm})$ on the temperature profile. It is observed that the temperature increases with an increase in $\mathrm{Gm}$. The effect of the 
Schmidt number $(\mathrm{Sc})$ on the temperature is depicted in Fig. 9. It is observed that the temperature decreases with an increase in Sc. The effect of the chemical reaction parameter $(K r)$ is depicted in Fig.10. It is observed that velocity decreases with an increase in the chemical reaction parameter $(\mathrm{Kr})$.

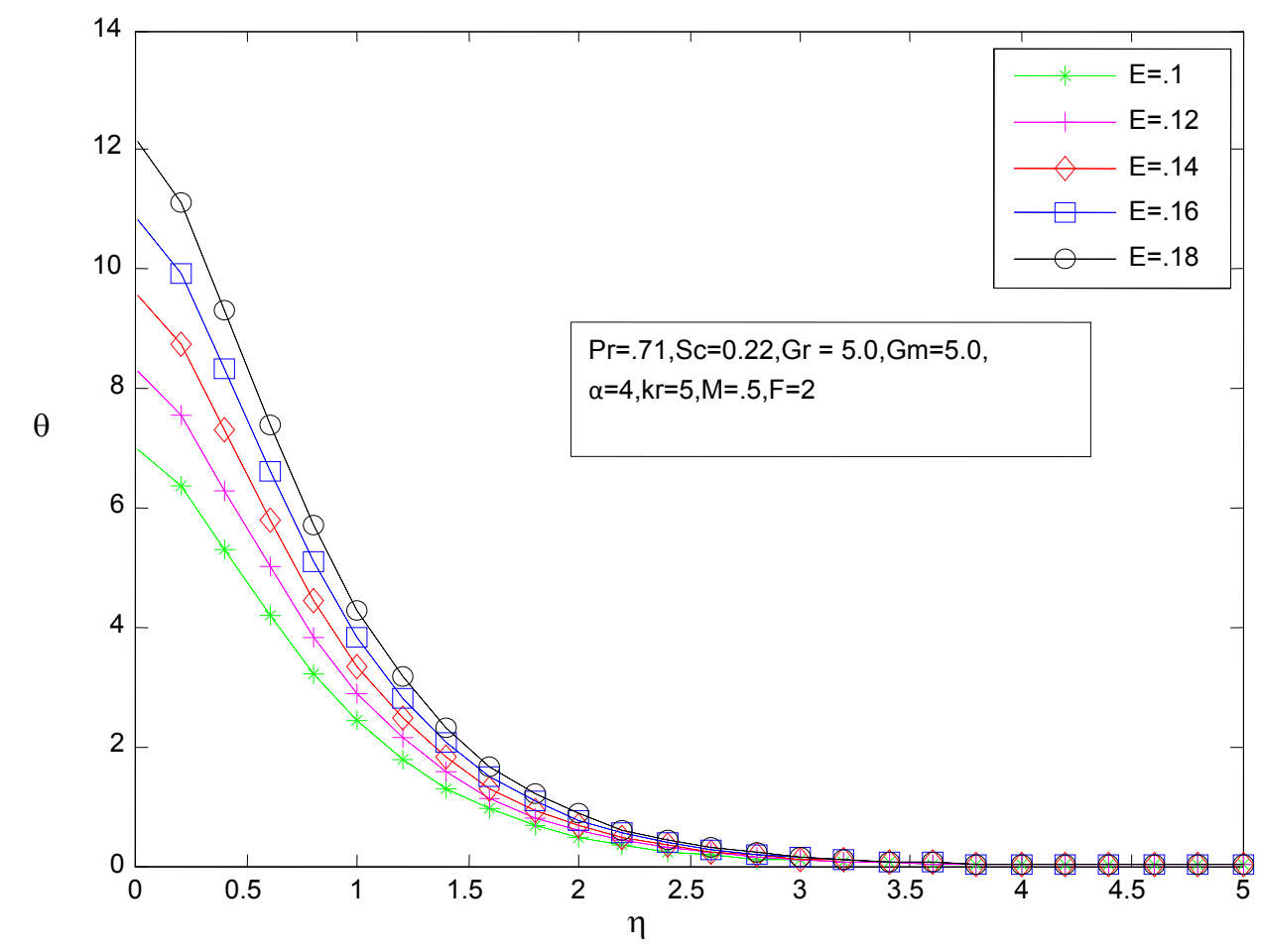

Fig.7. Temperature profile for different values of the Eckert number (E).

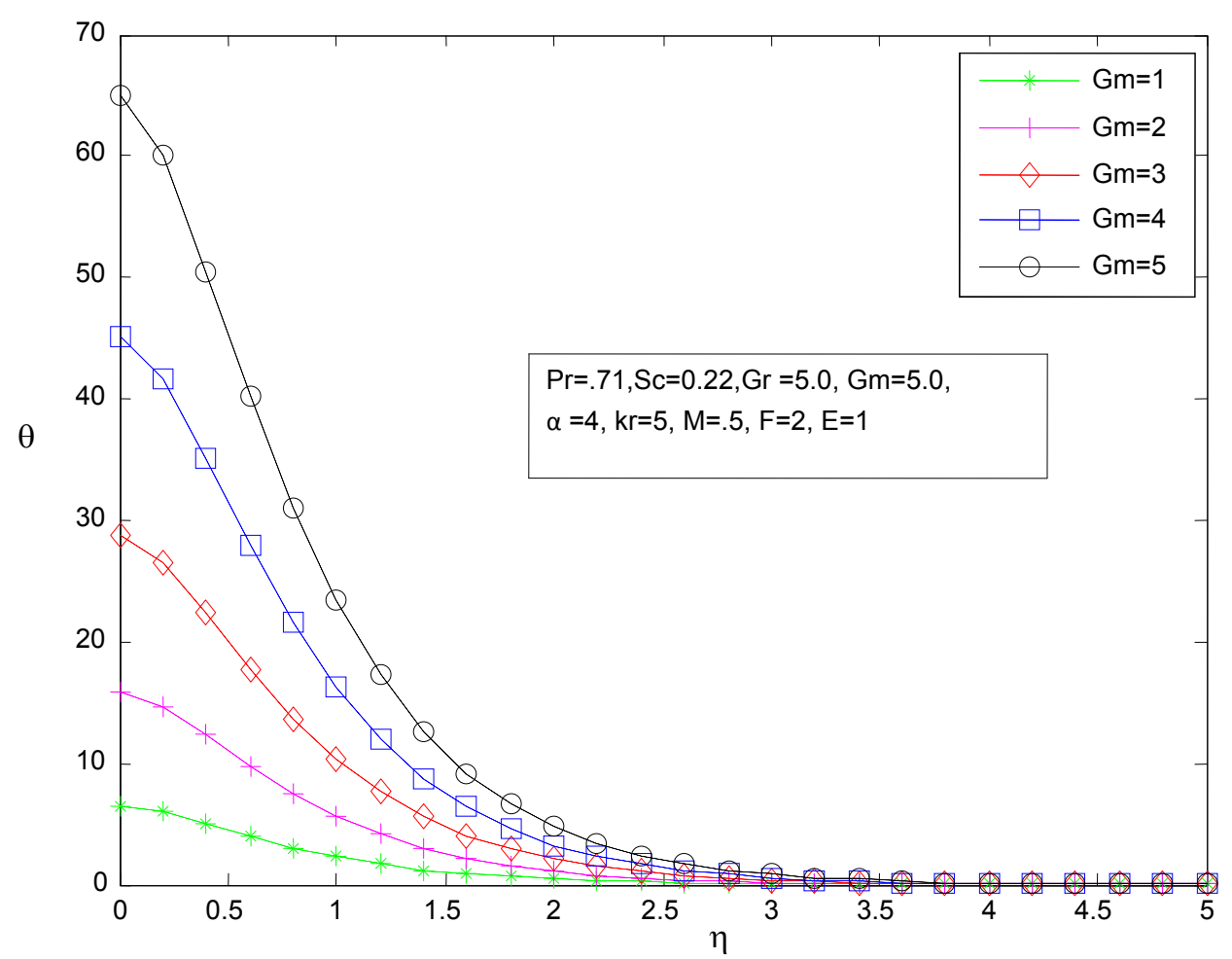

Fig.8. Temperature profile for different values of the $(\mathrm{Gm})$ Grashof number for mass transfer. 


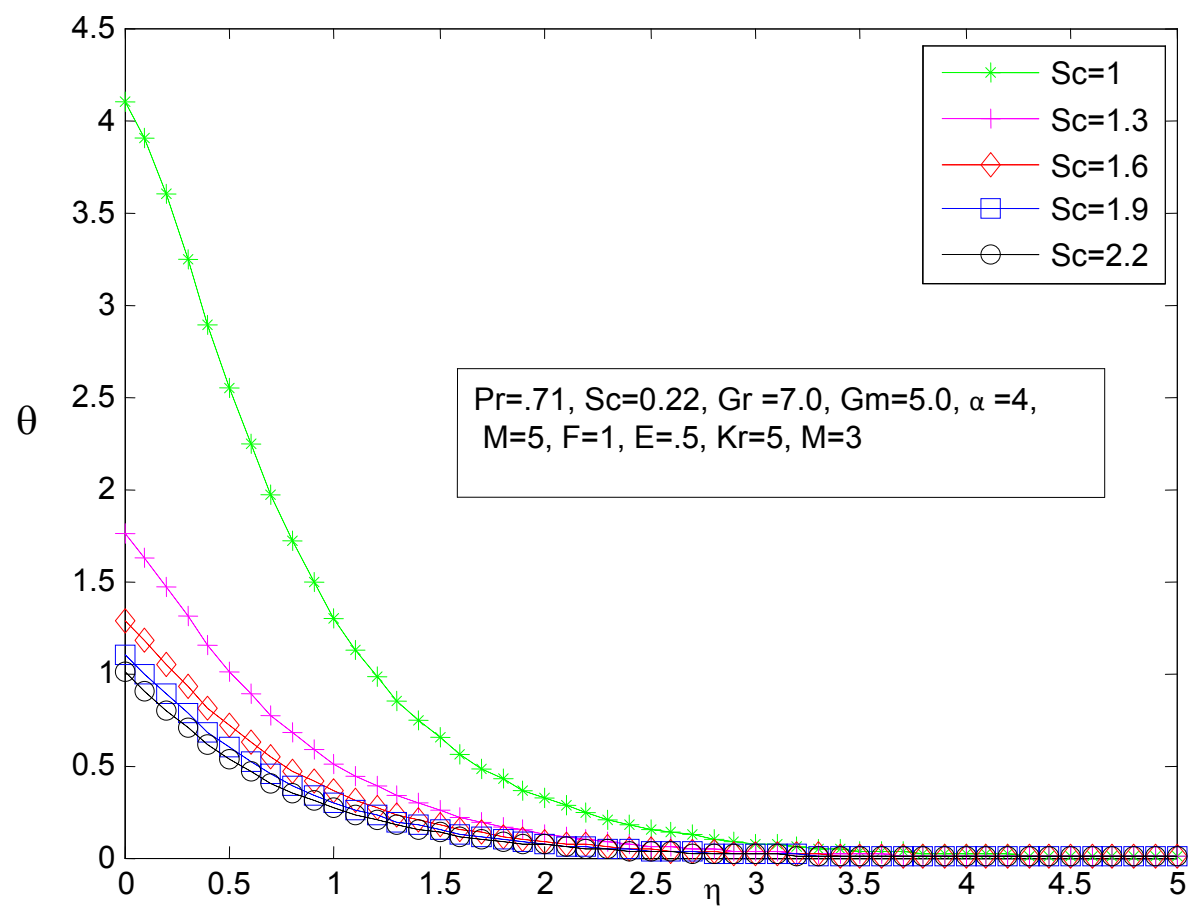

Fig.9. Temperature profile for different values of the Schmidt number ( $\mathrm{Sc}$ ).

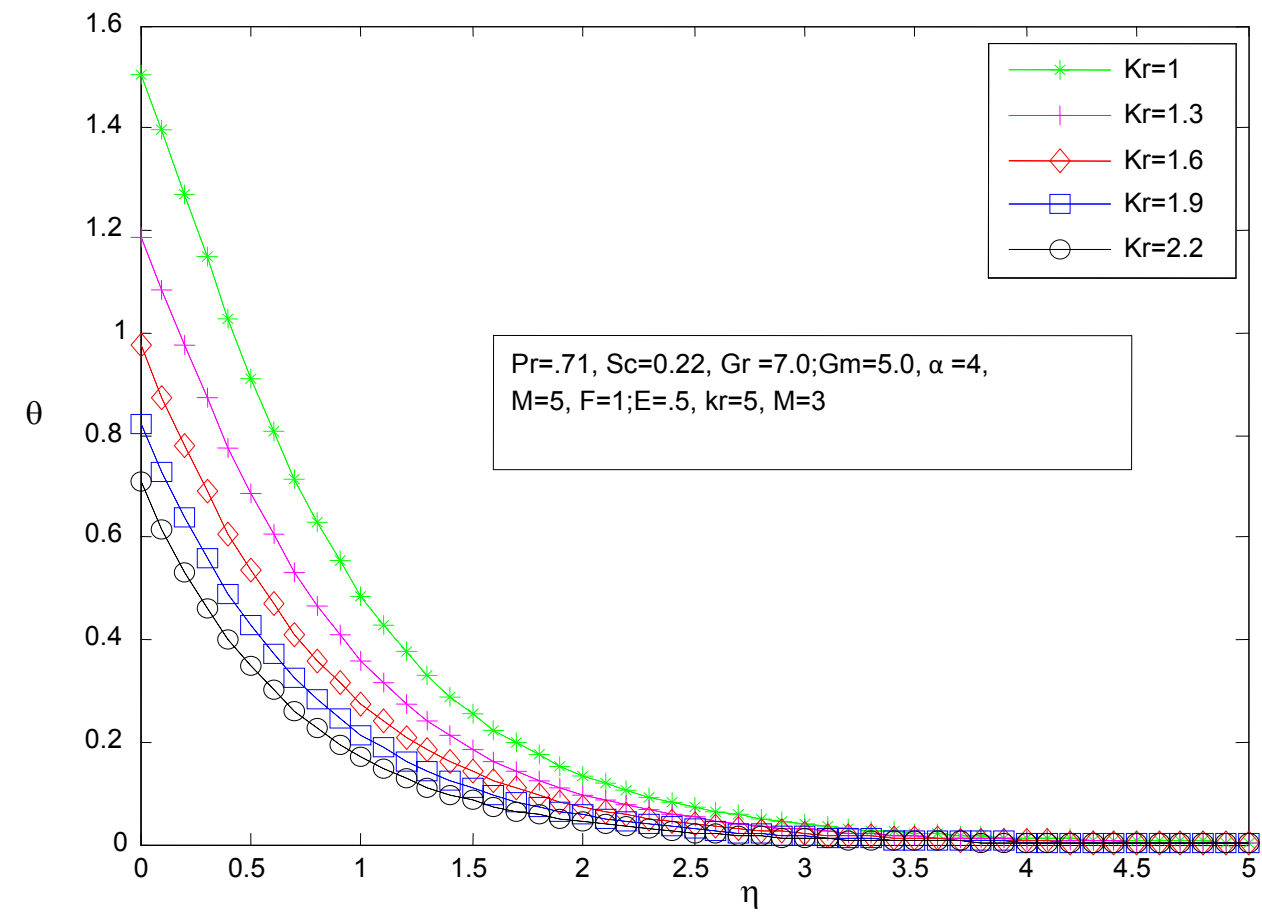

Fig.10. Temperature profile for different values of the chemical reaction parameter $(K r)$.

Concentration Profile: The concentration profiles are depicted in Figs 11-12. The effect of the Schmidt number $(\mathrm{Sc})$ on the concentration is presented in Fig.11. It is noted that the concentration decreases with an 
increase in the Schmidt Number ( Sc). In Fig.12, the effect of the chemical reaction parameter $(\mathrm{Kr})$ on the concentration is presented. It is seen that the concentration decreases with an increase in the chemical reaction parameter $(\mathrm{Kr})$.

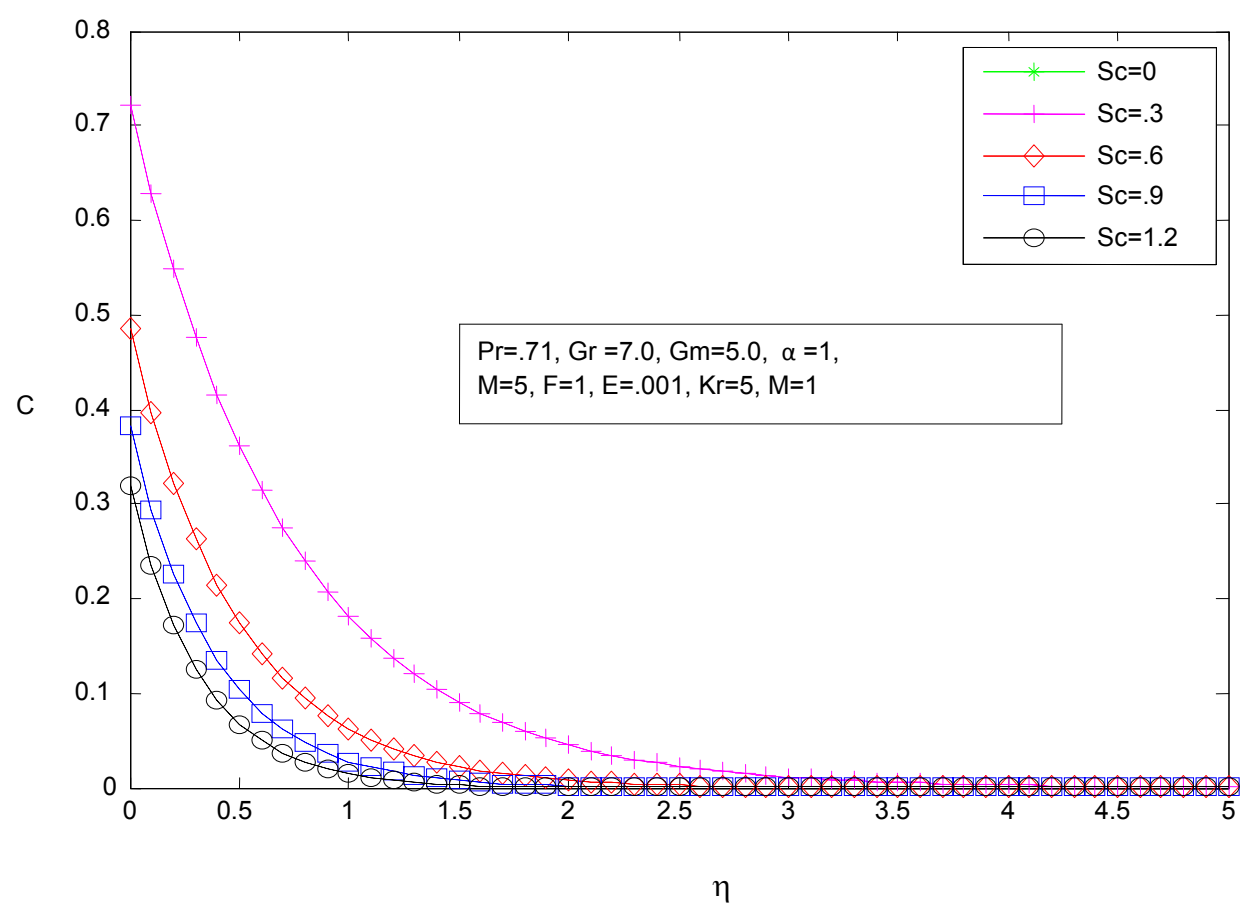

Fig.11. Concentration profile for different values of the Schmidt number (Sc).

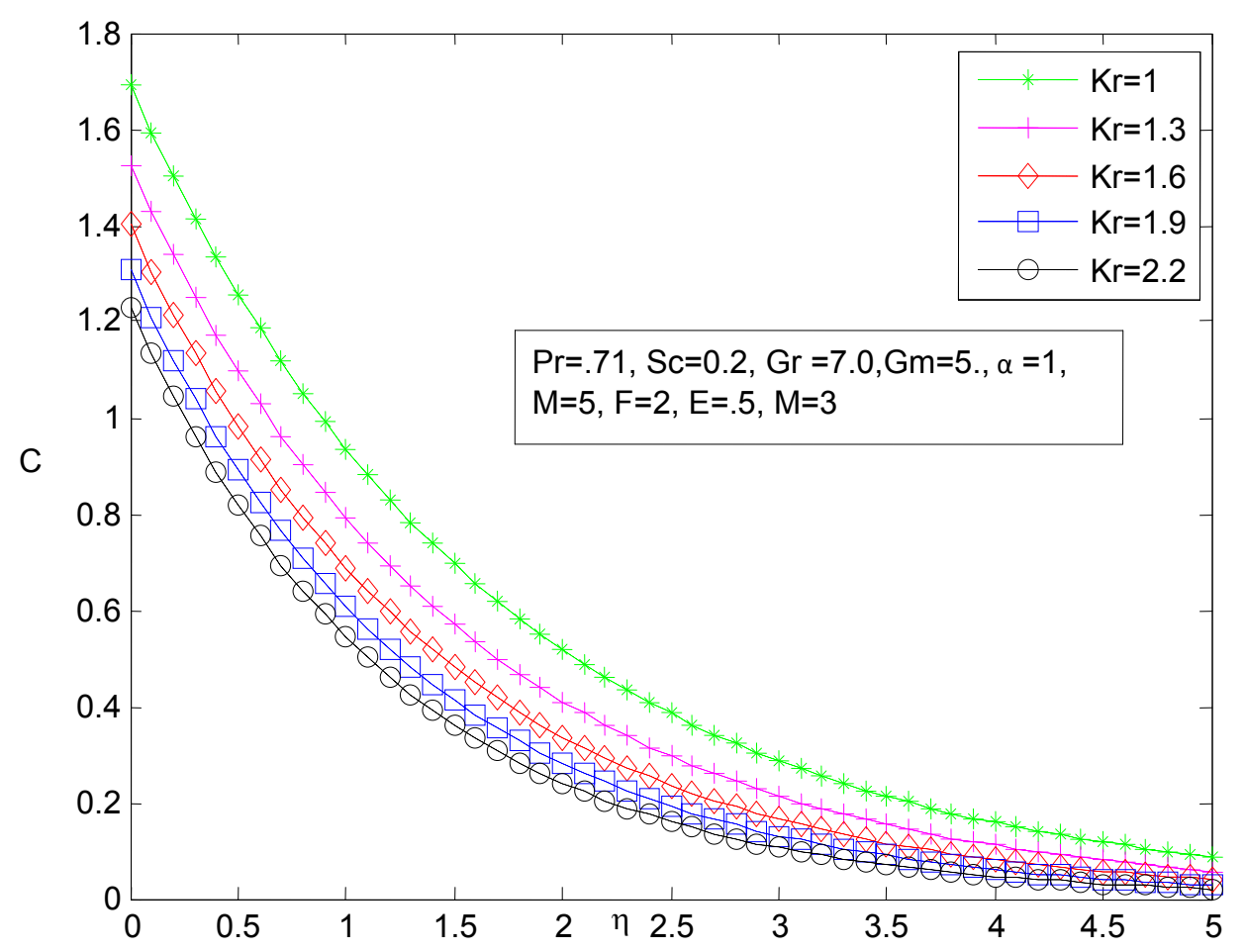

Fig.12. Concentration profile for different values of the chemical reaction parameter $(\mathrm{Kr})$. 


\section{Conclusions}

The governing equations for the unsteady MHD convective heat and mass transfer flow past a semiinfinite vertical permeable plate embedded in a porous medium with the radiation parameter and in the presence of a chemical reaction effect have been formulated. The plate is maintained at constant velocity and the flow is subjected to a transverse magnetic field. The governing partial differential equations were transformed into ordinary differential equations and analytical solutions have been derived using the perturbation method.

1. It was found that the velocity of the fluid flow increases with an increase in the permeability coefficient of a porous medium $(\alpha)$, radiation parameter $(F)$, Eckert number (E), Prandtl number (Pr) and (Gr) Grashof number for heat transfer.

2. The velocity of the fluid flow decreases with an increase in the Grashof number for mass transfer $(\mathrm{Gm})$. The temperature of the fluid increases with an increase in the Eckert number (E) and Grashof number $(\mathrm{Gm})$.

3. The temperature of the fluid decreases with an increase in the chemical reaction parameter $(K r)$ and Schmidt number $(\mathrm{Sc})$.

4. The concentration of the fluid increases with a decrease in the Schmidt number (Sc) and chemical reaction parameter $(\mathrm{Kr})$.

5. The presence of the radiation effect caused an incense in the velocity of the fluid flow.

The present study of the fluid flow over a vertical surface can be used as the basis for many scientific and engineering applications in case of vertical surfaces.

\section{Nomenclature}

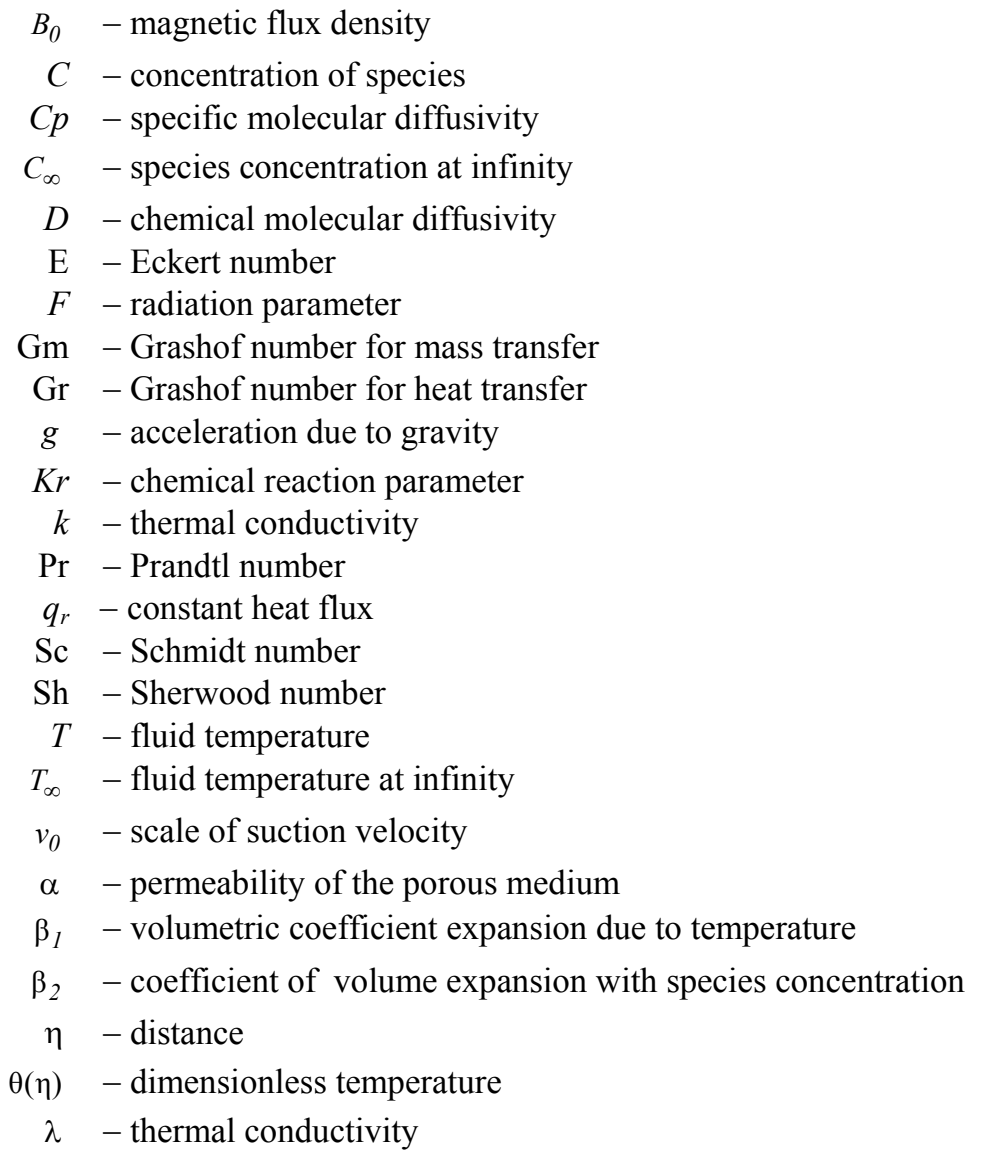


$\mu \quad-$ dynamic viscosity

$v \quad$ - kinematic viscosity

$\rho-$ density of the fluid

$\sigma \quad$ - electrical conductivity

$\tau-$ skin fraction

\section{Appendix}

$$
\begin{aligned}
& \delta=\frac{1}{\alpha}+M, \quad d_{1}=\frac{-\mathrm{Sc}+\sqrt{\mathrm{Sc}^{2}+4 \mathrm{Sc} k_{r}}}{2}, \quad d_{2}=\frac{-\mathrm{Sc}-\sqrt{\mathrm{Sc}^{2}+4 \mathrm{Sc} k_{r}}}{2}, \\
& e_{1}=\frac{-\operatorname{Pr}+\sqrt{\operatorname{Pr}^{2}+4 F}}{2}, \quad e_{2}=\frac{-\operatorname{Pr}-\sqrt{\operatorname{Pr}^{2}+4 \mathrm{~F}}}{2}, \\
& h_{1}=\frac{-1+\sqrt{1+4 \delta}}{2}, \quad h_{2}=\frac{-1-\sqrt{1+4 \delta}}{2} \\
& k_{1}=\frac{\mathrm{Gr}}{e_{2}\left[e_{2}^{2}+e_{2}-\delta\right]}, \quad k_{2}=\frac{\mathrm{Gm}}{d_{4}\left[d_{4}^{2}+d_{2}-\delta\right]}, \quad k_{3}=-\left(k_{1}+k_{2}\right), \\
& k_{4}=-\frac{\operatorname{Pr} h_{2}{ }^{2} k_{3}{ }^{2}}{\left[\left(2 h_{2}\right)^{2}+2 p_{r} h_{2}-F\right]}, \quad k_{5}=-\frac{p_{r} k_{1}{ }^{2} e_{2}{ }^{2}}{\left[\left(2 e_{2}\right)^{2}+2 p_{r} e_{2}-F\right]}, \\
& k_{6}=-\frac{p_{r} k_{2}^{2} d_{2}^{2}}{\left[\left(2 d_{2}\right)^{2}+2 p_{r} d_{2}-F\right]}, \quad k_{7}=-\frac{2 p_{r} k_{1} k_{3} h_{2} e_{2}}{\left[\left(e_{2}+h_{2}\right)^{2}+p_{r}\left(e_{2}+h_{2}\right)-F\right]}, \\
& k_{10}=-\frac{1}{e_{2}}\left[2 h_{2} k_{4}+2 e_{2} k_{5}+2 k_{6} d_{2}+k_{7}\left(h_{2}+e_{2}\right)+k_{8}\left(d_{2}+e_{2}\right)+k_{9}\left(h_{2}+d_{2}\right)\right] \text {, } \\
& k_{11}=-\frac{\mathrm{Gr} k_{10}}{\left[\left(e_{2}\right)^{2}+e_{2}-\delta\right]}, \quad k_{11}=-\frac{\mathrm{Gr} k_{4}}{\left[\left(2 h_{2}\right)^{2}+2 h_{2}-\delta\right]}, \quad k_{13}=-\frac{\mathrm{Gr} k_{5}}{\left[\left(2 e_{2}\right)^{2}+2 e_{2}-\delta\right]}, \\
& k_{14}=-\frac{\mathrm{Gr} k_{6}}{\left[\left(2 d_{2}\right)^{2}+2 d_{2}-\delta\right]}, \quad k_{15}=-\frac{\mathrm{Gr} k_{7}}{\left[\left(e_{2}+h_{2}\right)^{2}+p_{r}\left(e_{2}+h_{2}\right)-\delta\right]}, \\
& k_{16}=-\frac{\mathrm{Gr} k_{8}}{\left[\left(e_{2}+d_{2}\right)^{2}+p_{r}\left(e_{2}+d_{2}\right)-\delta\right]} \quad k_{17}=-\frac{\mathrm{Gr} k_{9}}{\left[\left(d_{2}+h_{2}\right)^{2}+\left(d_{2}+h_{2}\right)-\delta\right]}, \\
& k_{18}=-\left[k_{11}+k_{12}+k_{13}+k_{14}+k_{15}+k_{16}+k_{17}\right] .
\end{aligned}
$$




\section{References}

[1] Lin H.T. and Wu C.M. (1995): Combined heat and mass transfer by laminar natural convection from a vertical plate.- Heat and Mass Transfer, vol.30, pp.369-376.

[2] Chamkha A.J. (2000): Thermal radiation and buoyancy effects on hydro magnetic flow over an accelerating permeable surface with heat source or sink. - Int. J. Heat Mass Transfer, vol.38, pp.1699-1712.

[3] Chamkha A.J. (2004): Unsteady MHD convective heat and mass transfer past a semi-infinite vertical permeable moving plate with heat absorption. - Int. J. of Engg. Science, vol.42, pp.217-230.

[4] Chaudhary R.C., Sharma and B.K. and Jha A.K. (2006): Radiation effect with simultaneous thermal and mass diffusion in MHD mixed convection flow from a vertical surface with Ohmic heating. - Rom. Journ. Phys., vol.51, No.7-8, pp.715-727.

[5] Chaudhary R.C. and Jha A.K. (2008): Heat and mass transfer in elastico-viscous fluid past an impulsively started infinite vertical plate with hall effect. - Latin American Applied Research, vol.38, pp.17-26.

[6] Soundalgekar V.M. (1972): Viscous dissipation effects on unsteady free convective flow past an infinite vertical porous plate with constant suction. - International Journal of Heat Mass Transfer, vol.15, No.6, pp.1253-1261.

[7] Cortell R. (2006): Effects of viscous dissipation and work done by deformation on the MHD flow and heat transfer of a viscoelastic fluid over a stretching sheet. - Phys. Lett. A, vol. 357 pp.298-305.

[8] Kumar H. (2009): Radiative heat transfer with hydromagnetic flow and viscous dissipation over a stretching surface in the presence of variable heatflux. - Thermal Sci., vol.13, pp.163-169.

[9] Kishan N. and Amrutha P. (2010): Effects of viscous dissipation on MHD flow with heat and mass transfer over a stretching surface with heat source, thermal stratification and chemical reaction. - J. Naval Archit. Marine Eng., vol.7 pp.11-18.

[10] Sujon N. and Parveen N. (2014): Effects of viscous dissipation and heat generation on magneto hydrodynamics natural convection flow along a vertical wavy surface. - American Journal of Applied Mathematics, vol.2, No.6, pp.197-203.

[11] Hossain A.M., Alim M.A. and Rees D.A.S. (1999): Int. J. Heat Mass Transfer, vol.42, pp.181-191.

[12] Makinde O.D. (2005): Free convection flow with thermal radiation and mass transfer past a moving vertical porous plate. - Int. Comm. Heat Mass Transfer, vol.32, No.10, pp.1411-1419.

[13] Kulkarni P.S. and Patil P.M. (2008): Effects of chemical reaction on free convective flow of a polar fluid through a porous medium in the presence of internal heat generation. - Int. J. Therm. Sci., vol.47, No.8, pp.1043-1054.

[14] Chambre P.L. and Young J.D. (1958): On the diffusion of a chemically reactive species in a laminar boundary layer flow. - Phys. Fluids Flow, vol.11, pp.48-54.

[15] Chen T.S. and Yuh C.F. (1980): Combined heat and mass transfer in natural convection along vertical cylinder. International Journal of Heat Mass Transfer, vol.23, No.4, pp.451-461.

[16] Das U.N., Dekha R.K. and Soundalgekar V.M. (1999): Effect of mass transfer on flow past an impulsively started infinite vertical plate with constant heat flux and chemical reaction. - The Bulletin, GUMA, vol.5, pp.13-20.

[17] Muthucumarswamy R. (2002): Effects of chemical reaction on a moving isothermal vertical surface with suction. - Acta Mech., vol.155, pp.65-70.

[18] Seddek M.A. (2005): Finite-element method for the effects of chemical reaction, variable viscosity, thermophoresis and heat generation/absorption on a boundary-layer hydro magnetic flow with heat and mass transfer over a heat surface. - Acta Mech., vol.177, pp.1-18.

[19] Raptis A. and Perdikis C. (2006): Viscous flow over a non-linearly stretching sheet in the presence of a chemical reaction and magnetic field. - Int. J. Non-Linear Mech., vol.41, pp.527-529. 
[20] El-Kabeir S.M. and Abdou M. (2007): Chemical reaction, heat and mass transfer on MHD flow over a vertical isothermal cone surface in micropolar fluids with heat generation/absorption. - Appl. Math. Sci., vol.1, No.34, pp.1663-1674.

[21] Sudheer Babu M. and Satyanarayana P.V. (2009): Effects of the chemical reaction and radiation absorption on free convection flow through porous medium with variable suction in the presence of uniform magnetic field. Journal of Heat and Mass Transfer, vol.3, pp.219-234.

[22] Mahapatra N., Dash G.C., Panda S. and Acharya M. (2010): Effects of chemical reaction on free convection flow through a porous medium bounded by a vertical surface. - Journal of Engineering Physics and Thermophysics, vol.83, No.1, pp.130-140.

[23] Chandra Shekar B. and Kishan N. (2015): Radiation effects on unsteady MHD convective heat and mass transfer past a vertical plate with chemical reaction and viscous dissipation. - Alexandria Engineering Journal, vol.54, pp.661-671.

[24] Ibrahim S.M. and Suneetha K. (2016): Effects of thermal diffusion and chemical reaction on MHD transient free convection flow past a porous vertical plate with radiation, temperature gradient dependent heat source in slip flow regime. - Journal of Computational and Applied Research in Mechanical Engineering, vol.5, No.2, pp.8395.

[25] Yamamoto K. and Iwamura N. (1976): Flow with convection acceleration through a porous medium. - J. Eng. Math., vol.10, No.1, pp.41-54.

[26] Raptis A. and Singh A.K. (1985): Free convection flow past an impulsively started vertical plate in a porous medium by finite difference method. - Astrophys. Space Sci., vol.112, pp.259-265.

[27] Sattar M.A., Rahman M.M. and Alam M.M. (2000): Free convection flow and heat transfer through a porous vertical flat plate immersed in a porous medium. - J. Energy Res., vol.22, No.1, pp.17-21.

[28] Samad M.A. and Rahman M.M. (2006): Theramal radiation interaction and unsteady MHD flow past a vertical porous plate immersed in a porous medium. - Journal of Naval Architechture and Marine Engineering, vol.3, No.2, pp.7-14.

[29] Sharma P.R. and Singh G. (2008): Unsteady MHD free convective flow and heat transfer along a vertical porous plate with variable suction and internal heat generation. - Int. J. Appl. Math and Mech., vol.4, pp.1-8.

[30] Sharma P.R., Dadkeech I.K. and Singh G. (2012): Heat and mass transfer effects on unsteady MHD free convective flow along a vertical porous plate with internal heat generation and variable suction. - Int. J. of Math Archive, vol.3, No.5, pp.2163-2172.

[31] Cogley A.C., Vincent W.G. and Giles S.E. (1968): Differential approximation to radiative heat transfer in a nongrey gas near equilibrium. - AIAA J, vol.6, pp.551-553.

[32] Shercliff J.A. (1965): A Text Book of Magnetohydrodynamics. - London: Pergamon Press.

[33] Cramer K.P. and Pai S.I. (1973): Magneto Fluid Dynamics for Engineers and Applied Physics. - New York: McGraw-Hill Book Co..

[34] Eckert E.R.G. and Drake R.M. (1979): Heat and Mass Transfer. - New Delhi: Tata McGraw-Hill, 2nd Ed.. 